\title{
All-Fiber, Single-Mode Spectral Beam Combining of High Power Tm-Doped Fiber Lasers
}

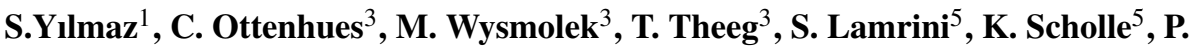 \\ Fuhrberg $^{5}$, H. Sayınc ${ }^{3}$, F. Ö. İlday ${ }^{1,2}$, J. Neumann ${ }^{3}$, L. Overmeyer ${ }^{4}$, and D. Kracht ${ }^{3}$. \\ ${ }^{1}$ Department of Physics, Bilkent University, Ankara, 06800, Turkey \\ ${ }^{2}$ Department of Electrical and Electronics Engineering, Bilkent University, Ankara, 06800, Turkey \\ ${ }^{3}$ Laser Zentrum Hannover e. V., Hollerithallee 8, 30419 Hannover, Germany \\ ${ }^{4}$ Institut für Transport und Automatisierungstechnik, An der Universität 2, 30823 Garbsen, Germany \\ ${ }^{5}$ Lisa Laser Product, OHG, Max-Planck-Str 1, 37191 Katlenburg-Lindau, Germany \\ sinem.yilmaz@fen.bilkent.edu.tr
}

\begin{abstract}
Signal beam combining of Tm-doped fiber lasers can increase the laser output power while simultaneously maintaining the single mode beam quality. We demonstrate an all-fiber integrated dual-wavelength Tm-doped fiber laser with an output power of $36 \mathrm{~W}$ by using the spectral beam combining method. The constituent lasers are operating at the wavelengths 1949 and $1996 \mathrm{~nm}$ and an in-house-made WDM is used for combination of these two different wavelengths. All-fiber truly single mode power combining is demonstrated for the first time in this wavelength region.
\end{abstract}

\section{Introduction}

High power fiber lasers with high beam quality have numerous applications in many scientific and technological fields. Among them, thulium-doped fiber lasers are a newly emerging, but rapidly expanding field and a top choice for eye-safe high power applications with their central wavelength around $2 \mu \mathrm{m}$. In addition, Tm-doped fiber lasers around $2 \mu \mathrm{m}$ are ideally suited for nonlinear frequency conversion into the mid-IR, which is important for a range of spectroscopy, medical and defense applications. Despite the natural technical difficulties associated with a newly developing technological frontier, Tm-doped fiber lasers appear to be well-suited for the generation of high signal power with a low quantum defect and subsequently a low thermal load due to an efficient cross-relaxation process which occurs in heavly doped thulium fibers, making such fibers suitable for high power lasers [1].



Fig. 1. One of the continuous-wave fiber oscillator setups for spectral-beam combining and schematic diagram of the spectral beam-combining setup. HR-FBG: High reflective fiber-Bragg grating, LR-FBG: Low reflective fiber-Bragg grating, PLS: Pump-light stripper, WDM: Wavelengthdivision multiplexer.

Scaling lasers to high average output power while maintaining single-spatial mode beam profiles is of current interest $[2,3]$. Nevertheless, scaling the power of single mode fiber lasers is limited by optical or thermal damage of the fiber, thermal aberrations and nonlinear effects. A promising power scaling approach for fiber lasers is spectral beam combining. In such a scheme, distinct laser sources, which are overlapped by using fiber components, are suitable 
for handling high power. All fiber beam combining of sources employing core diameters of $15 \mu \mathrm{m}$ was demonstrated before in the wavelength range around $2 \mu \mathrm{m}$ [4]. However, the brilliance of the laser sources degraded completely due to the use of combiners employing multimode fibers. Spectral beam combining utilizing single mode WDMs is a promising approach, as the high beam quality of the laser building blocks is maintained. Typically, WDMs consist of two fibers enabling the combination of two wavelengths per fiber component. We propose the use of a WDM cascade consisting of three WDMs. Two of them are used for combining lasers with a small wavelength separation of approximately $20 \mathrm{~nm}$ and a third one is used to combine both wavelengths groups. The cascading concept offers maximum efficiency for largest possible separation of the wavelengths groups (limited by gain bandwidth) and smallest possible separation of wavelengths within respective groups (limited by manufacturing parameters of WDMs). In our contribution, we demonstrate truly single mode power combining with a first WDM capable of handling high power.

\section{Experimental Setup}

We are using an in-house-made WDM for spatially combining signals and scaling up the power available from a single mode fiber. The experimental setup of the fiber laser system and spectral-beam combination part are shown in Fig. 1. It consists of two identical continuous wave oscillators each emitting narrow linewidth signals at two distinct wavelengths which are $1949 \mathrm{~nm}$ and $1996 \mathrm{~nm}$. The oscillator is pumped with a fiber coupled pump diode at $793 \mathrm{~nm}$ and the pump light is coupled into the cavity by using pump combiners. The active fiber has a length of $5 \mathrm{~m}$ with a core/cladding of $10 / 130 \mu \mathrm{m}$ and has a pump light absorption of $3 \mathrm{~dB} / \mathrm{m}$. The cavity consists of fiber-Bragg gratings as highly reflective mirror and output coupler. Constituent oscillators are optimized with respect to maximum output power. Then, inhouse-made pump-light strippers (PLS) are used at the output of these oscillators for eliminating unabsorbed pump light while maintaining the beam quality of individual ports. A pure signal power of $22 \mathrm{~W}$ is obtained from the output of each oscillators after eliminating a pump power of $8 \mathrm{~W}$ from each oscillator.

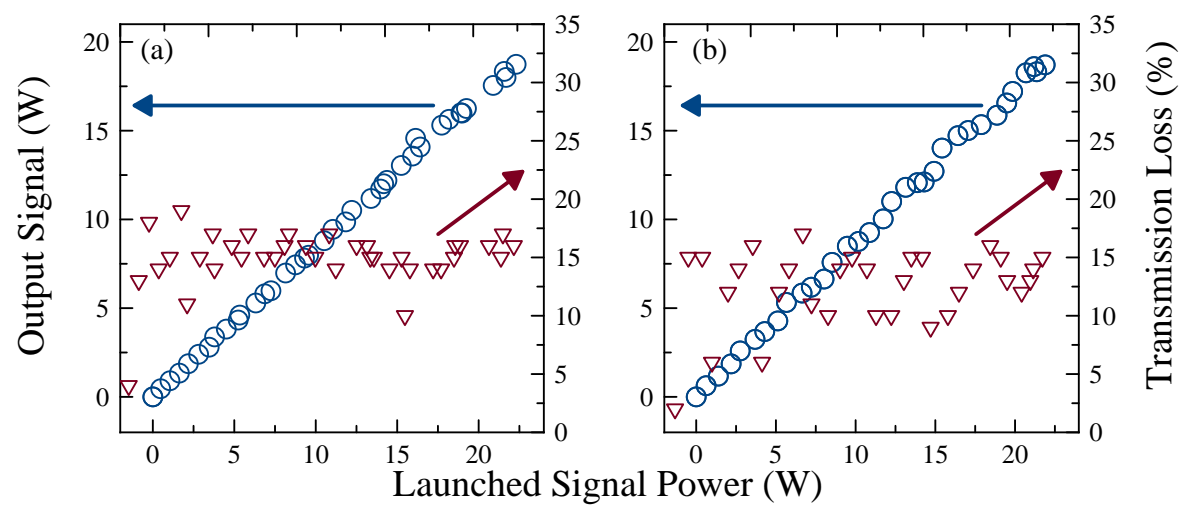

Fig. 2. Optical power of the combined beam at the signal output port of the WDM and transmission loss with respect to the launched signal power for (a) $1950 \mathrm{~nm}$ and (b) $1997 \mathrm{~nm}$.

\section{Results}

The lasers are spliced to the in-house-made WDM for spatially overlapping the beams with differing wavelengths as shown in Fig. 1. First, the behaviour of the constituent lasers are investigated separately from the signal output port of the WDM. The measured output power as a function of the launched signal power for both of the lasers is shown in Fig. 2. Additionally, the transmission loss of the WDM is indicated in Fig. 2 and the coupling efficiency of the WDM is almost the same for both wavelengths which is approximately $85 \%$.

Combination of signals by the WDM is also analyzed simultaneously in Fig. 3. The combined laser power with respect to the launched signal power is shown in Fig 3(d). We achieved more than $36 \mathrm{~W}$ of output power at a total input power of $44 \mathrm{~W}$, which results in a combining efficiency of $80 \%$. Degradation of the coupling efficiency could be caused by coupling of the $3 \mathrm{~W}$ signal power to the idler port due to heating of the WDM. The spectrum of the combined beam is shown in Fig. 3(a). Two narrow linewidth peaks can be observed and zoomed versions of these spectra are shown in Fig. 3(b)-(c).

Our approach for achieving a truly single-mode laser source based on power combination in the wavelength range around $2 \mu \mathrm{m}$ enables further power scaling by increasing the output power of the distinct laser oscillators and indepen- 
dently from that by using a cascaded combination scheme of WDMs. The utilized WDM will be a building block of a WDM cascade in future work. Maximizing the output power of the used laser oscillators around $2 \mu \mathrm{m}$ and minimizing the losses of the WDM will enable scaling the output power by a factor of four with respect to a sinle oscillator.
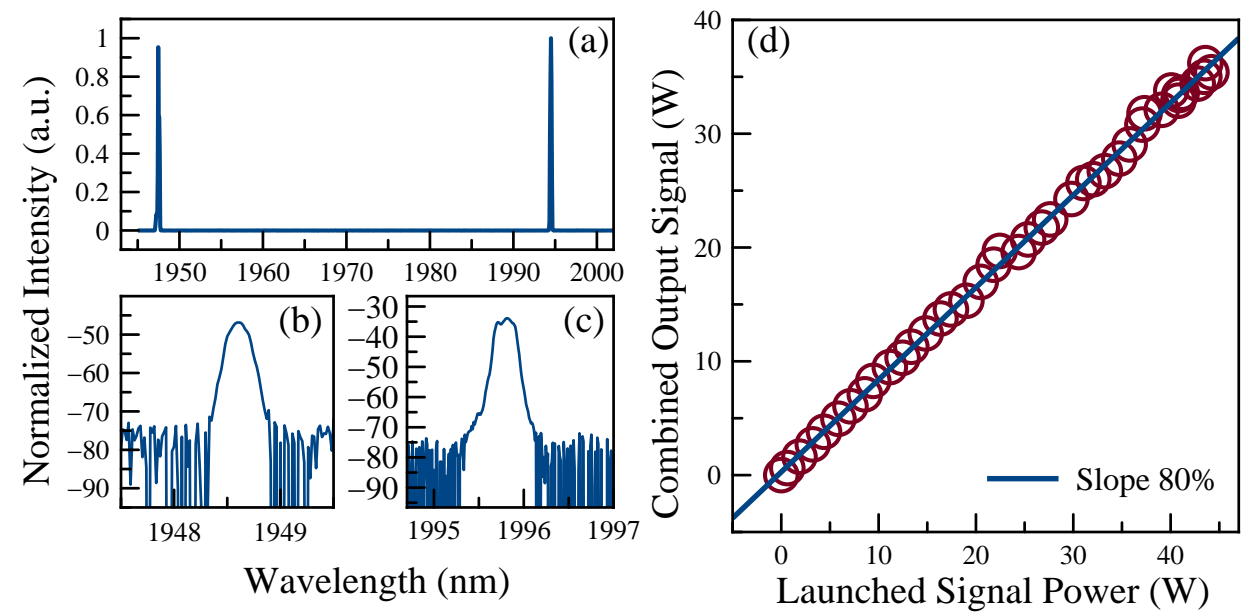

Fig. 3. (a) Spectral composition of the combined beam measurement. (b) Spectrum of single oscillator system at $1948 \mathrm{~nm}$ and (c) $1996 \mathrm{~nm}$. (d) Output characteristics of the spectral-beam combining setup with $80 \%$ combining efficiency.

\section{Summary}

In summary, we have demonstrated all-fiber truly single mode power combination of Tm-doped fiber lasers. By using fiber-Bragg gratings as a highly reflective mirror and output coupler, the distinct fiber lasers operate at wavelengths of 1949 and $1996 \mathrm{~nm}$. The wavelengths are only determined by the FBGs. The lasers are combined by using an in-house-made WDM. The expected wavelength spacing can be freely specified by choosing FBG pairs with proper central wavelength and can be combined by changing the specifications of WDM. We achieved more than $36 \mathrm{~W}$ of output power and the combining efficiency is measured to be $80 \%$. As the mode field of the laser radiation in the two distinct laser oscillators, the pump-light stripper and the WDM have a diameter of approximately $11 \mu \mathrm{m}$, truly single mode power combining is demonstrated for the first time around the wavelength range of $2 \mu \mathrm{m}$ and with a continuing improvement in high power fiber lasers and WDMs with high power handling capability, single mode operation at higher output power level can be generated. The demonstrated power combining scheme also offers a new perspective for high brightness pump sources for Holmium doped fiber lasers.

Acknowldgement: The authors thank the German Research Foundation (DFG) for funding the SFB/TRR 123 Planare Optronische Systeme (PlanOS).

\section{References}

1. S. D. Jackson " Cross relaxation and energy transfer upconversion processes relevant to the functioning of $2 \mu \mathrm{m}$ Tm-doped silica fibre lasers," Optics Communications, 14, pp. 197-203 (2004).

2. G. D. Goodno, L. D. Book, and J. E. Rothenberg, "Low-phase-noise, single-frequency single-mode $608 \mathrm{~W}$ thulium fiber amplifier," Optics Letters, 34, pp. 1204 (2009).

3. K. Yin, B. Zhang, G.Xue, L. Li, and J. Hou, "High-power all-fiber wavelength-tunable thulium doped fiber laser at $2 \mu \mathrm{m}$ " Optics Express, 22, pp. 19947 (2014).

4. A. Hemming, N. Simakov, A. Davidson, S. Bennetts, M. Hughes, N. Carmody, P. Davies, L. Corena, D. Stepanov, J. Haub, R. Swain, and A. Carter. "A Monolithic cladding pumped holmium-doped fibre laser," CLEO: Science and Innovation, San-Jose, California United States, ISBN:978-1-55752-972-5 (2013). 